\title{
Therapeutic efficacy and artemisinin resistance in northern Myanmar: evidence from in vivo and molecular marker studies
}

Moe Kyaw Myint ${ }^{1}$, Charlotte Rasmussen ${ }^{2}$, Aung Thi ${ }^{3}$, Dorina Bustos ${ }^{4}$, Pascal Ringwald ${ }^{2}$ and Khin Lin ${ }^{1 *}$

\begin{abstract}
Background: In Myanmar, three types of artemisinin-based combination therapy (ACT) are recommended as first-line treatment of uncomplicated falciparum malaria: artemether-lumefantrine (AL), artesunate-mefloquine $(A S+M Q)$, and dihydroartemisinin-piperaquine (DP). Resistance to both artemisinins and ACT partner drugs has been reported from the Greater Mekong Sub-region, and regular efficacy monitoring of the recommended ACT is conducted in Myanmar. This paper reports on results from studies to monitor the efficacy of the three forms of ACT in sentinel sites in northern Myanmar, and investigations of mutations in the Kelch13 (k13) propeller domain.

Methods: Seven therapeutic efficacy studies were conducted in 2011-12 and 2014 in three sentinel sites in Myanmar (Tamu, Muse, Tabeikkyin). Three studies were done for the evaluation of AL (204 patients), two studies for AS + MQ (119 patients) and two studies for DP (147 patients). These studies were done according to 2009 standard WHO protocol. Polymorphisms in the 13 propeller domain were examined in dried blood spots collected on day 0. The primary endpoint was adequate clinical and parasitological response (ACPR) on day 28 for AL and on day 42 for $\mathrm{DP}$ and $\mathrm{AS}+\mathrm{MQ}$, corrected to exclude re-infection using polymerase chain reaction (PCR) genotyping. Safety data were collected through self-reporting.
\end{abstract}

Results: PCR-corrected ACPR was $97.2-100 \%$ for AL, $98.6-100 \%$ for AS + MQ and 100\% for DP across the study sites and years. All studies found a prevalence of $k 13$ mutations (>440) above $23 \%$ in the day-0 samples. The F446I mutation was the most common mutation, making up $66.0 \%$ of the mutations found. Seven out of nine day-3 positive patients were infected with $k 13$ wild type parasites. The remaining two cases with day-3 parasitaemia had the P574L mutation.

Conclusions: The efficacy of AL, AS + MQ and DP remains high in northern Myanmar despite widespread evidence of $k 13$ mutations associated with delayed parasite clearance. This study showed that already in 2012 there was a high frequency of $k 13$ mutations in Myanmar on the border with India. The high efficacy of the recommended ACT gives confidence in the continued recommendation of the use of these treatments in Myanmar.

Trial registration numbers ACTRN12611001245987 (registered 06-12-2011) and ACTRN12614000216617 (registered 28-02-2014)

Keywords: Myanmar, Treatment efficacy, Plasmodium falciparum, Molecular markers, k13

\footnotetext{
*Correspondence: dr.khinlin.dir@gmail.com

${ }^{1}$ Department of Medical Research (Pyin Oo Lwin Branch), Ministry

of Health and Sports, Pyin Oo Lwin 05062, Myanmar

Full list of author information is available at the end of the article
} 


\section{Background}

In 2014, Myanmar reported 152,195 malaria cases, $72 \%$ of them due to Plasmodium falciparum. Overall, this is a $74 \%$ reduction from the number of cases reported in 2010 [1]. A key factor in this significant reduction of malaria in Myanmar has been expanded access to artemisinin-based combination therapy (ACT). Currently, there are three ACT recommended as first-line treatment for P. falciparum: artemether-lumefantrine (AL), artesunate-mefloquine (AS + MQ) and dihydroartemisinin-piperaquine (DP) [2]. A decline in ACT efficacy has been seen in other countries in the Greater Mekong Sub-region (GMS), and could pose a threat to continued progress towards malaria elimination [3].

Myanmar is the only country in the GMS with more than one recommended first-line ACT in a given geographical area. While $\mathrm{AL}$ is the treatment that is most frequently used in the public sector and by most international non-governmental organizations, the other ACT are available in the private sector, making monitoring of all three recommended ACT a necessity. Therapeutic efficacy studies (TES) for anti-malarial medicine are the gold standard, providing the information needed to guide treatment policies. Monitoring of molecular markers for resistance can provide supplementary information and is helping to improve the surveillance of resistance.

All ACT contain an artemisinin and a partner drug. Resistance to artemisinin was first identified in clinical studies in 2006 close to the Cambodia-Thailand border. However, retrospective analysis of molecular markers indicates that artemisinin resistance likely emerged as early as 2001. Artemisinin resistance is defined as delayed parasite clearance following treatment with an artesunate monotherapy or with an ACT. In TES, this is measured by the proportion of patients found positive on day 3 after treatment [4].

Artemisinin resistance has been shown to be associated with point mutations in the propeller region of the $P$. falciparum Kelch13 (k13) gene [5]. At present, 108 different non-synonymous $k 13$ mutations have been reported, and these different mutations can have varying effect on the clearance genotype. Studies have shown a clear distinction between the $k 13$ mutations that are most frequent in eastern GMS (Cambodia, Lao PDR, Vietnam) and western GMS (China, Myanmar, Thailand) [4].

In Myanmar, delayed parasite clearance in patients treated with an ACT was first seen in 2009 in southern Myanmar [6]. Recent studies have shown high prevalence of $k 13$ mutations extending through much of Myanmar $[7,8]$. However, artemisinin resistance and delayed parasite clearance will not necessarily lead to treatment failure as TES of ACT for treatment of falciparum malaria have continued to show high efficacy. In the GMS, high treatment failure rates following treatment with an ACT have mostly been observed where concomitant resistance to the partner drug exists [4].

This paper reports on the results of seven therapeutic ACT efficacy studies of the three recommended ACT in 2011-12 and 2014 in northern Myanmar, and results from investigations of mutations in the $k 13$ propeller domain.

\section{Methods \\ Study design and sites}

Two studies were conducted in September 2011-September 2012 to evaluate AL and AS + MQ in Muse in northern Myanmar, close to the border with China. In Tamu, close to the Indian border, two studies were done in June-October 2012 to evaluate AL and AS + MQ. Three additional studies were done in May-November 2014: two in Tabeikkyin to evaluate AL and DP, and one in Tamu to evaluate DP. Location of the sites is shown in Fig. 1.

The study design was an open-label, one-arm, prospective evaluation of clinical and parasitological responses to directly observed treatment for uncomplicated malaria. The WHO protocol for monitoring therapeutic efficacy was used [9]. In the sites where two different ACT were assessed, the treatments were studied sequentially so that recruitment of patients for the assessment of one ACT was completed before patients were recruited for assessment of a second ACT.

Estimation of the target sample size in each study was based on an assumption of treatment failure of $5 \%$, with a $95 \%$ confidence level and precision of $5 \%$. Allowing for a $10 \%$ loss to follow-up, the target sample size in each study was 80 cases. Ethical approvals were obtained from the Ethics Review Committee of the Department of Medical Research, Yangon, Myanmar, and the WHO Ethics Review Committee, Geneva, Switzerland. The studies were registered at Australian New Zealand Clinical Trials Registry [10].

\section{Study population}

Cases were enrolled after obtaining a patient's, or parent's/guardian's written consent. Eligible patients were aged six years and above (excluding females $12-17$ years) with microscopically confirmed, uncomplicated $P$. falciparum mono-infection, a parasite density of 500-100,000 asexual parasites $/ \mu \mathrm{l}$, and a body temperature $\geq 37.5{ }^{\circ} \mathrm{C}$, or a history of fever during the previous $24 \mathrm{~h}$. Pregnant women were excluded from the study, as were patients with signs of severe malaria or with febrile conditions due to diseases other than malaria. 


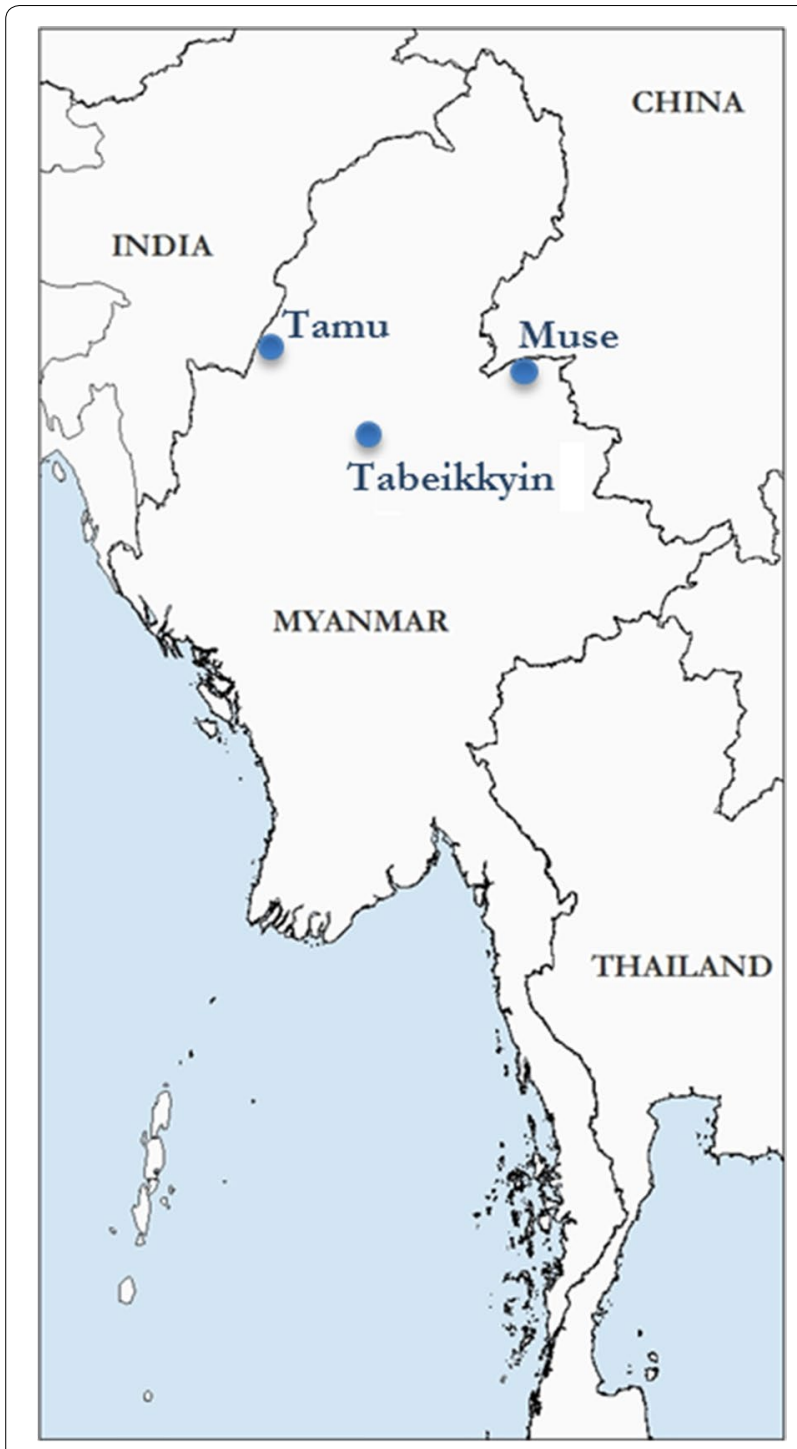

Fig. 1 Map showing location of study sites

\section{Treatment}

AL (Coartem ${ }^{\circledR}$, Novartis, Switzerland) was administered at a target dose of $1.3 / 8 \mathrm{mg} / \mathrm{kg}$ twice daily for 3 days. For treatment with AS + MQ, artesunate (Guilin Pharmaceutical Ltd, China) was given at a target dose of $4 \mathrm{mg}$ / $\mathrm{kg}$ once a day for 3 days. Mefloquine (Mepha Ltd, Switzerland) was given at a target dose of $15 \mathrm{mg} / \mathrm{kg}$ once on the first day followed by $10 \mathrm{mg} / \mathrm{kg}$ on the second day. DP (Duo-Cotecxin ${ }^{\circledR}$, Zhejiang Holley Nanhu Pharmaceutical Ltd, China) was given at a target dose of 2-2.4/16$19.2 \mathrm{mg} / \mathrm{kg}$ once a day for 3 days. Quality-controlled medications for the studies were provided by WHO Global Malaria Programme.

\section{Patient follow-up}

Patients received medications under direct observation on the first 3 days. Clinical and parasitological followup was done on days $0,1,2,3,7,14,21$, and 28 for AL, and additionally on days 35 and 42 for AS + MQ and DP. Any adverse events reported by patients were recorded at each visit. Monitoring included axillary temperature, parasitaemia and gametocytaemia.

\section{Laboratory methods}

Thick and thin blood films were obtained from each patient at screening. Blood films were also obtained on days $1,2,3,7,14,21,28$, (and 35 and 42 for AS + MQ or $\mathrm{DP})$, and any other day if the patient returned spontaneously and parasitological re-assessment was needed. The blood smears were stained with fresh Giemsa, and examined at a magnification of $1000 \times$ to identify parasite species and determine parasite density. Asexual parasitaemia was determined from Giemsa-stained thick blood smears against the number of parasites per 200 white blood cells on day 0 , based on an assumed density of 6000 white blood cells per $\mu \mathrm{l}$ of blood. Gametocytes were similarly enumerated. A blood smear was declared negative after examination of 1000 white blood cells [9]. Quality control of microscopy for all studies was done by an external consultant from the Research Institute for Tropical Medicine in the Philippines, a WHO Collaborating Centre for malaria diagnosis.

Dried blood spots were obtained for polymerasechain reaction (PCR) at enrolment (day 0) and on follow-up days 7, 14, 21, and 28 (and 35 and 42 for AS + MQ or DP). PCR genotyping was performed on paired dried blood spots in the case of parasitaemia detected on or after day 7 to distinguish between recrudescence and re-infection. This PCR genotyping determined polymorphisms in merozoite surface protein-1 ( $m s p-1)$, merozoite surface protein-2 $(m s p-2)$ and glutamate-rich protein (glurp) polymorphism, as per WHO methods [9]. The PCR testing was done at the laboratory of Department of Medical Research, Yangon, Myanmar.

Polymorphisms in the $k 13$ propeller domain were examined in the dried blood spots collected on day 0 . The method used was a nested PCR protocol followed by Sanger sequencing using primers specific to $P$. falciparum. The amplicon used for sequencing covered $740 \mathrm{bp}$, which included the $k 13$ propeller domain [11]. The sequencing was done at Faculty of Tropical Medicine, Mahidol University, Bangkok, Thailand, and at the Malaria Molecular Epidemiology Unit, Institut Pasteur, Phnom Penh, Cambodia. 


\section{Outcome evaluation and analysis}

Treatment outcomes were classified on the basis of an assessment of the parasitological and clinical outcome of anti-malarial treatment according to $\mathrm{WHO}$ guidelines. All patients were classified as having early treatment failure (ETF), late clinical failure (LCF), late parasitological failure (LPF), or an adequate clinical and parasitological response (ACPR) [9].

The primary outcome measure was PCR-corrected ACPR. The patients were excluded from the analysis if PCR results were not available to check for re-infection. If PCR results suggested re-infection, then the patient was excluded from the pre-protocol analysis.

Information on ACT-related side effects was collected through self-reporting and recorded in the case reporting forms. EpiData software was used for double data entry (and validation) and analysis (version 3.1 for entry and version 2.2.2.182 for analysis, EpiData Association, Odense, Denmark).

\section{Results}

Seven studies were conducted in three sentinel sites in Myanmar in 2011-2012 and 2014 to assess the efficacy of AL (three studies), AS + MQ (two studies) and DP (two studies). A total of 4919 patients were screened and 470 enrolled. Of these, 20 patients were lost to follow-up before day 28/42, three patients were withdrawn due to PCR-confirmed re-infection, one patient was excluded from the analysis with parasitaemia without PCR being able to determine re-infection or recrudescence, leaving 446 patients for evaluation at day 28/42 (Fig. 2).

\section{Baseline characteristics of enrolled patients}

The demographic and clinical characteristics of the study population in each site is shown in Table 1 . The majority of the enrolled patients in all studies were males (66.791.1\%). The mean age in the studies was between 22.8 and 39 years with the majority of the patients being above 15 years $(80-100 \%)$. The geometric mean of parasitaemia

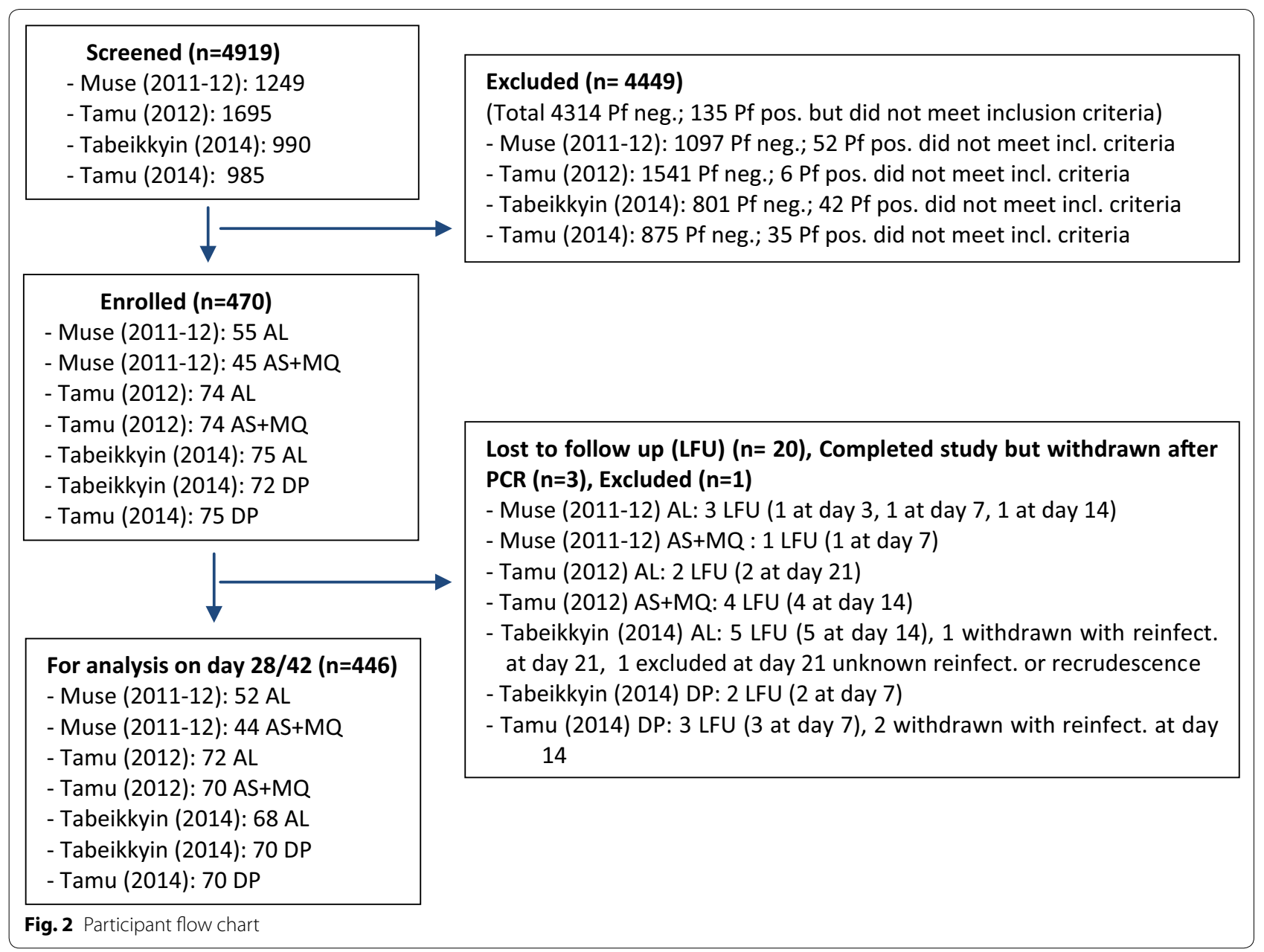


Table 1 Baseline characteristics of patients by study

\begin{tabular}{|c|c|c|c|c|c|c|c|c|}
\hline \multirow[t]{2}{*}{ Site } & \multirow[t]{2}{*}{ Year } & \multirow[t]{2}{*}{ Drug } & \multirow[t]{2}{*}{ Enrolled $n$} & \multirow{2}{*}{$\begin{array}{l}\text { Males } \\
\text { n (\%) }\end{array}$} & \multicolumn{2}{|l|}{ Age } & \multirow{2}{*}{$\begin{array}{l}\text { Weight mean kgs } \\
\text { (range) }\end{array}$} & \multirow{2}{*}{$\begin{array}{l}\text { Parasitaemia }(/ \mathrm{ml}) \\
\text { geometric mean } \\
\text { (range) }\end{array}$} \\
\hline & & & & & $\begin{array}{l}\text { Mean years } \\
\text { (range) }\end{array}$ & $\begin{array}{l}>15 \text { years } \\
\mathrm{n}(\%)\end{array}$ & & \\
\hline Muse & $2011-12$ & $\mathrm{AL}$ & 55 & $45(81.8)$ & $22.8(5-56)$ & $44(80)$ & $56.9(30-70)$ & $3745(1002-76,421)$ \\
\hline Muse & $2011-12$ & $\mathrm{AS}+\mathrm{MQ}$ & 45 & $41(91.1)$ & $29.6(16-65)$ & $45(100)$ & $59.4(50-65)$ & $3210(1000-54,436)$ \\
\hline Tamu & 2012 & $\mathrm{AL}$ & 74 & $64(86.5)$ & $33.2(11-60)$ & $71(95.9)$ & $55.8(30-70)$ & $3494(1002-31,492)$ \\
\hline Tamu & 2012 & $\mathrm{AS}+\mathrm{MQ}$ & 74 & $62(83.8)$ & $36.6(13-64)$ & $73(98.6)$ & $58(40-75)$ & $3774(1081-15,310)$ \\
\hline Tabeikkyin & 2014 & $A L$ & 75 & $52(69.3)$ & $36.6(8-60)$ & $74(98.7)$ & $52.9(30-65)$ & $4251(1524-12,244)$ \\
\hline Tabeikkyin & 2014 & $\mathrm{DP}$ & 72 & $60(83.3)$ & $35.6(12-65)$ & 71 (98.6) & $55(32-65)$ & $4408(1063-16,424)$ \\
\hline Tamu & 2014 & DP & 75 & $50(66.7)$ & $39(10-74)$ & $72(96.0)$ & $56.9(32-75)$ & $5199(2556-14,664)$ \\
\hline
\end{tabular}

at admission in the seven studies was $3210-5199$ asexual parasites $/ \mu$ l.

\section{Adverse events}

Overall, 27\% of all study participants reported one or more side effects (Table 2). With AS $+\mathrm{MQ}$, 33\% reported side effects, for AL $26 \%$ reported side effects, and for DP $22 \%$ reported side effects. The most frequent side effect by type of ACT was insomnia (10.9\%) with AS + MQ, dizziness (9.3\%) with $\mathrm{AL}$, and vomiting (6.8\%) with DP. No serious adverse events were reported.

\section{Clinical and parasitological responses}

Cases positive on day 3 were identified in three of the seven studies (Table 3). In total, only nine of the 470 enrolled patients were found to be positive on day 3. All the day- 3 positive cases were followed up to day $28 / 42$, and all had ACPR. The highest number of day- 3 positive cases was in the 2011-12 study in Muse monitoring efficacy of AL: 4 of $55(7.3 \%)$ patients were still positive at day 3 . Of the nine day-3 positive cases, eight were identified in studies for AL efficacy. The geometric mean of day 0 parasitaemia $(/ \mu \mathrm{l})$ among the nine day-3 positive cases

Table 2 Adverse events reported by treatment

\begin{tabular}{|c|c|c|c|c|}
\hline & Total $(n=470)$ & $\begin{array}{l}\text { Artemether }+ \\
\text { lumefantrine }(n=204)\end{array}$ & $\begin{array}{l}\text { Artesunate }+ \\
\text { mefloquine }(n=119)\end{array}$ & $\begin{array}{l}\text { Dihydroarte- } \\
\text { misinin + pipe- } \\
\text { raquine }(n=147)\end{array}$ \\
\hline & n (\%) & n (\%) & n (\%) & n (\%) \\
\hline \multicolumn{5}{|c|}{ Number of adverse events per patient } \\
\hline 0 & $345(73)$ & $150(74)$ & $80(67)$ & $115(78)$ \\
\hline 1 & $60(13)$ & $25(12)$ & $16(13)$ & $19(13)$ \\
\hline 2 & $64(14)$ & $29(14)$ & $22(19)$ & $13(9)$ \\
\hline 3 & $1(<1)$ & 0 & $1(<1)$ & 0 \\
\hline \multicolumn{5}{|c|}{ Most frequent type of adverse event reported } \\
\hline Dizziness & $30(6.4)$ & $19(9.3)$ & $9(7.6)$ & $2(1.4)$ \\
\hline Nausea & $29(6.2)$ & $13(6.4)$ & $8(6.7)$ & $8(5.4)$ \\
\hline Vomiting & $27(5.7)$ & $8(3.9)$ & $9(7.6)$ & $10(6.8)$ \\
\hline Loss of appetite & $19(4.0)$ & $9(4.4)$ & $3(2.5)$ & $7(4.8)$ \\
\hline Insomnia & $15(3.2)$ & $2(1.0)$ & $13(10.9)$ & 0 \\
\hline Palpitation & $14(3.0)$ & $11(5.4)$ & $2(1.7)$ & $1(0.7)$ \\
\hline Diarrhoea & $13(2.8)$ & $3(1.5)$ & $3(2.5)$ & $7(4.8)$ \\
\hline Abdominal pain & $10(2.1)$ & $3(1.5)$ & $7(5.9)$ & 0 \\
\hline Asthaenia & $10(2.1)$ & $1(0.5)$ & $4(3.4)$ & $5(3.4)$ \\
\hline Headache & $6(1.3)$ & $6(2.9)$ & 0 & 0 \\
\hline Cough & $6(1.3)$ & $2(1.0)$ & 0 & $4(2.7)$ \\
\hline Joint/muscle pain & $5(1.1)$ & $3(1.5)$ & $1(0.8)$ & $1(0.7)$ \\
\hline Skin rash & $3(0.6)$ & $2(1.0)$ & $1(0.8)$ & 0 \\
\hline Nightmare & $2(0.4)$ & 0 & $2(1.7)$ & 0 \\
\hline
\end{tabular}




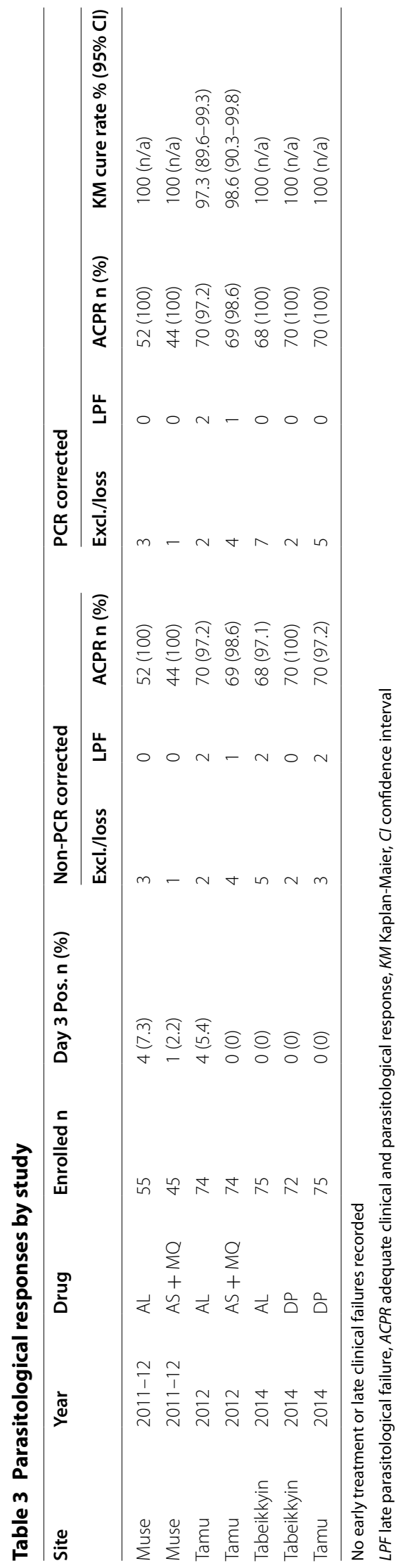


was 5962 (range 1079-31,492). At day 3, the geometric mean of parasitaemia $(/ \mu \mathrm{l})$ among the positive cases was 69 (range 23-131).

Table 3 shows parasitological responses for the different studies. The proportion of cases with ACPR was 97.1-100\% before PCR correction, and $97.2-100 \%$ after PCR correction. Three patients were classified as treatment failures after PCR correction and the exclusion of patients with re-infections. All three cases were identified with recrudescence on day 21 in the 2012 studies in Tamu: two of them in the study for AL, and the third in the AS + MQ study. None of the three patients with treatment failures was identified as having parasitaemia on day 3.

\section{k13 Genotyping}

The $k 13$ gene was successfully sequenced in 288 (61.3\%) of the 470 day- 0 samples. The main reason for failure to do sequencing was that for some patients too little blood was collected on the filter papers. Among the sequenced samples, the prevalence of $k 13$ mutations (>440) was $33.7 \%(97 / 288)$ (Table 4). The F446I mutation was the most common mutation, making up 66.0\% (64/97) of the mutations being present in $22.2 \%$ of the samples. The highest prevalence of $k 13$ mutations was in Thabeikkyin in 2014 (49.2\%).

For the three cases with treatment failure, $k 13$ sequencing was not possible. Sequencing showed that $k 13$ genotypes in seven of the nine cases with parasitaemia on day 3 were $k 13$ wild type parasites. The remaining two cases with day-3 parasitaemia had the P574L mutation. While the numbers are small, there is evidence $(\mathrm{p}<0.05$ using a Fisher's exact test) of significant differences in the proportion of day-3 positive cases by genotype.

\section{Discussion}

This paper reports on the therapeutic efficacy of all three recommended first-line ACT from sentinel sites in northern Myanmar. The efficacy of all ACT in the studies were high $(>97 \%)$ in both PCR uncorrected and corrected analyses, meeting the $\mathrm{WHO}$ recommendation that cure rates for falciparum malaria should be at least 90\% [9]. These results are similar to results found in other studies in northern Myanmar [12, 13].

A total of eight different $k 13$ mutations (position $>440$ ) were recorded in the studies. Artemisinin resistance was first identified in southern Myanmar close to the border with Thailand where the most extensive data are from. In the 2012 study in Tamu on the Myanmar-India border, $k 13$ mutations were found in $28.9 \%$ of the samples, mainly the F446I mutation (25 of $28 k 13$ mutations). These are the earliest data documenting $k 13$ mutations in this part of Myanmar.
Data from the Indian side of the border show that AL is highly efficacious (Mishra and Valecha, pers. comm.). AL was introduced as first-line treatment in northeastern India after increasing treatment failures with artesunate + sulfadoxine-pyrimethamine (AS + SP) due to resistance to SP. Data from 2010-2013 showed very low prevalence of $k 13$ mutations in India. Four non-synonymous $k 13$ mutations were identified among 384 samples but only at very low frequencies $(0.26 \%, 1 / 384)$. Of these four mutations, three (G533A, R561H and A578S) were detected in the northeastern states [14]. Of these three mutations, only the R561H were identified in Tamu and only in the 2014 study.

The most common mutation in all the Myanmar study sites was the F446I. In Tamu in 2012, the prevalence of F446I was $25.8 \%$. This remained nearly constant with the prevalence of F446I in Tamu in 2014 at $24.6 \%$. One mutation in Tamu not found in 2012, but found in seven patients (12.3\%) in 2014 was the $\mathrm{R} 561 \mathrm{H}$ mutation. In Tabeikkyin in 2014, the N458Y mutation was identified in seven patients (11.1\%). Both the F446I and the R561H mutations have been reported in other studies from northern Myanmar [7, 12, 15]. The N458Y mutation has previously been identified in southern and central Myanmar [7]. Two highest prevalent mutations found in Muse in Myanmar-China border in 2011-2012 were the F446I and P574L. This finding is similar to other study done in that border area [15]. The C580Y mutation was not found in the samples. However, other study done in southern part of Myanmar found C580Y mutation in Kayin State, Myanmar-Thailand Border [7]. This mutation is common in the eastern part of the GMS, becoming increasingly dominant among $k 13$ mutations in Cambodia [16].

The prevalence of molecular markers for artemisinin resistance found in the studies has not resulted in a high prevalence of day-3 positivity, or a fall in efficacy of the ACT. The majority (7/9) of the day-3 positive cases were $k 13$ wild type parasites. The overall percentage of patients positive on day 3 is only $3.1 \%$. However, among patients identified as having the P574L mutation, 33\% (2/6) was found to have day-3 parasitaemia. Despite the low number of patients, there is evidence of significant differences $(\mathrm{p}<0.05)$ in the proportion of day-3 positive cases by genotype.

The overall low proportion of day- 3 positive patients could be for several reasons. The patients had significant lower parasitaemia on day 0 than in other research studies done in the area, which makes it less likely that parasites would be found on day 3 , even if there are relatively slow parasite clearance rates. These studies do not allow for estimation of parasite clearance half-life as blood was collected only once a day rather than every 6 or $8 \mathrm{~h}$. Another possible explanation for the low proportion of 


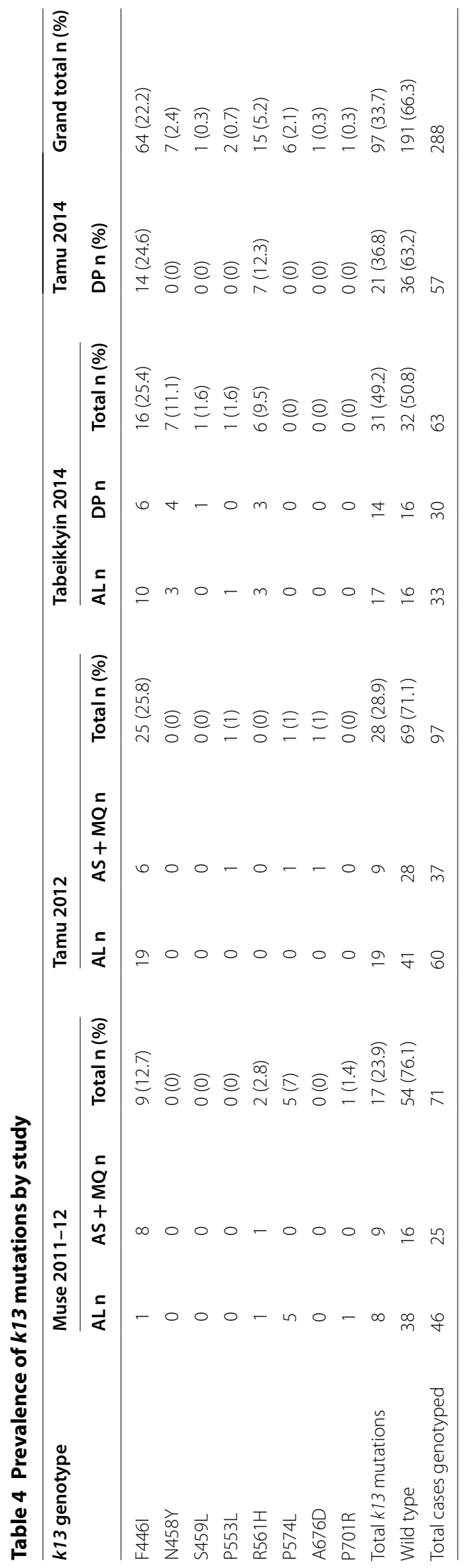


cases positive on day 3 is the prevalence of $k 13$ mutation. The F446I mutation may be associated with only an intermediate artemisinin resistance phenotype, not resulting in a slowing of the parasite clearance to the same extent as mutations most prevalent in eastern parts of the GMS [12]. In addition, factors such as immunity and pharmacokinetics may have played a role.

A high efficacy was found for all the three recommended ACT, giving confidence in the continued recommendation of these ACT as appropriate first-line treatments for $P$. falciparum. So far studies done in all sentinel site of Myanmar showed high level of efficacy $(>90 \%)$ to the three recommended ACT. The role of triple ACT for treatment of uncomplicated falciparum malaria can be determined only after the completion of ongoing ACT versus triple ACT studies in Myanmar. Nevertheless, continued monitoring will be needed. While there is no evidence for $\mathrm{ACT}$ partner drug resistance resulting in ACT failures in these studies, partner drug resistance is leading to ACT failures in other GMS countries, emphasizing the risks and the need to continue monitoring ACT efficacy.

Reporting of adverse events showed that among patients receiving $\mathrm{AS}+\mathrm{MQ}$, there was a slightly higher proportion of patients reporting one or more adverse events. This could affect the effectiveness of the treatment, as a higher proportion of patients on AS + MQ may choose to discontinue treatment before completion of the full three-day treatment.

\section{Conclusion}

All the three recommended ACT in Myanmar have been shown to be highly efficacious despite widespread evidence of $k 13$ mutations associated with delayed parasite clearance after treatment with an artemisinin. This study showed that already in 2012 there was a high frequency of $k 13$ mutations in Myanmar on the border with India. The high efficacy of the recommended ACT gives confidence in the continued recommendation to use these treatments in Myanmar. The threat of resistance to ACT partner drugs resulting in falling ACT efficacy elsewhere in the region highlights the need for continual monitoring of ACT efficacy.

\footnotetext{
Authors' contributions

$\mathrm{DB}, \mathrm{KL}, \mathrm{MKM}$, and PR developed the protocols. $\mathrm{KL}$ and $M K M$ were involved in data acquisition. $\mathrm{KL}, \mathrm{MKM}$ and $\mathrm{CR}$ analysed the data. $\mathrm{DB}, \mathrm{KL}, \mathrm{MKM}$, and $\mathrm{PR}$ provided study oversight. MKM and CR interpreted the data and drafted the manuscript. All authors had access to the study data, contributed to the paper. All authors read and approved the final manuscript.

\section{Author details}

${ }^{1}$ Department of Medical Research (Pyin Oo Lwin Branch), Ministry of Health and Sports, Pyin Oo Lwin 05062, Myanmar. ${ }^{2}$ World Health Organization, 20 Avenue Appia, 1211 Geneva 27, Switzerland. ${ }^{3}$ National Malaria Control Programme, Ministry of Health and Sports, Nay Pyi Taw, Myanmar. ${ }^{4}$ World Health Organization, Bangkok, Thailand.
}

\section{Acknowledgements}

The authors would like to express their appreciation to all of the study patients, and to the parents and guardians of the children who participated. Support for the development of the manuscript was provided through the Structured Operational Research and Training Initiative (SORT IT), a global partnership led by the Special Programme for Research and Training in Tropical Diseases at the World Health Organization (WHO/TDR). SORT IT programmes include a teaching component developed jointly by the International Union Against Tuberculosis and Lung Disease (The Union) and Medécins sans Frontières. The specific SORT IT programme which resulted in this manuscript was implemented by: WHO/TDR, The Department of Medical Research (DMR), Ministry of Health, Myanmar, The National Malaria Control Programme and The National TB Programme, Department of Health, Ministry of Health, Myanmar; the WHO Country Office for Myanmar, the Operational Research Unit (LuxOR), Médecins Sans Frontières, Brussels Operational Centre, Luxembourg; the Centre for Operational Research, The Union, France; The Institute of Tropical Medicine, Antwerp, Belgium; the Academic Model Providing Access to Healthcare (AMPATH), Kenya; and, the National Institute of Parasitic Diseases, CDC, China.

\section{Competing interests}

The authors declare that there is no competing interests regarding the publication of this paper. DB, CR and PR are staff members of the World Health Organization. DB, CR and PR are solely responsible for the views expressed, and they do not necessarily represent decisions, policies or views of the World Health Organization.

\section{Availability of data and materials}

The datasets generated during and/or analysed during the current study are available in the WHO database.

\section{Ethics approval and consent to participate}

Ethical approvals were obtained from the Ethics Review Committee of the Department of Medical Research, Yangon, Myanmar, and the WHO Ethics Review Committee, Geneva, Switzerland. Patients were enrolled only after obtaining the patient's, or parent's/guardian's informed consent.

\section{Funding}

These studies were supported by Bill and Melinda Gates Foundation and USAID via the World Health Organization, Global Malaria Programme, Geneva, Switzerland, and World Health Organization, Southeast Asia Regional Office, New Delhi, India.

\section{Publisher's Note}

Springer Nature remains neutral with regard to jurisdictional claims in published maps and institutional affiliations.

Received: 3 January 2017 Accepted: 14 March 2017

Published online: 07 April 2017

\section{References}

1. WHO. World malaria report 2015. Geneva: World Health Organization; 2015.

2. Myanmar Ministry of Health. National malaria treatment guidelines. Nay Pyi Taw: Department of Health, Ministry of Health; 2009.

3. WHO. Strategy for malaria elimination in the Greater Mekong Subregion (2015-2030). Geneva: World Health Organization; 2015.

4. WHO. Artemisinin and artemisinin-based combination therapy resistance. Status report. Geneva: World Health Organization; 2016. http://www.who. int/malaria/publications/atoz/update-artemisinin-resistance-april2016/ en/. Accessed 28 Jun 2016.

5. Ashley EA, Dhorda M, Fairhurst RM, Amaratunga C, Lim P, Suon S, et al. Spread of artemisinin resistance in Plasmodium falciparum malaria. N Engl J Med. 2014;371:411-23.

6. Kyaw MP, Nyunt MH, Chit K, Aye MM, Aye KH, Aye MM, et al. Reduced susceptibility of Plasmodium falciparum to artesunate in southern Myanmar. PLoS ONE. 2013;8:e57689. 
7. Tun KM, Imwong M, Lwin KM, Win AA, Hlaing TM, Hlaing T, et al. Spread of artemisinin-resistant Plasmodium falciparum in Myanmar: a cross-sectional survey of the K13 molecular marker. Lancet Infect Dis. 2015;15:415-21.

8. Win AA, Imwong M, Kyaw MP, Woodrow CJ, Chotivanich K, Hanboonkunupakarn B, et al. K13 mutations and pfmdr1 copy number variation in Plasmodium falciparum malaria in Myanmar. Malar J. 2016;15:110.

9. WHO. Methods for surveillance of antimalarial drug efficacy. Geneva: World Health Organization; 2009.

10. Australian New Zealand Clinical Trials Registry. http://www.anzctr.org.au

11. Ariey F, Witkowski B, Amaratunga C, Beghain J, Langlois A-C, Khim N et al. A molecular marker of artemisinin-resistant Plasmodium falciparum malaria. Nature. 2014;505:50-5.

12. Tun KM, Jeeyapant $A$, Imwong $M$, Thein M, Aung SSM, Hlaing TM, et al. Parasite clearance rates in upper Myanmar indicate a distinctive artemisinin resistance phenotype: a therapeutic efficacy study. Malar J. 2016;15:185.
13. Liu H, Yang HL, Tang LH, Li XL, Huang F, Wang JZ, et al. In vivo monitoring of dihydroartemisinin-piperaquine sensitivity in Plasmodium falciparum along the China-Myanmar border of Yunnan Province, China from 2007 to 2013. Malar J. 2015;14:47.

14. Mishra N, Prajapati SK, Kaitholia K, Bharti RS, Srivastava B, Phookan S, et al. Surveillance of artemisinin resistance in Plasmodium falciparum in India using the kelch13 molecular marker. Antimicrob Agents Chemother. 2015;59:2548-53.

15. Wang Z, Shrestha S, Li X, Miao J, Yuan L, Cabrera M, et al. Prevalence of K13-propeller polymorphisms in Plasmodium falciparum from ChinaMyanmar border in 2007-2012. Malar J. 2015;14:168

16. Leang R, Canavati SE, Khim N, Vestergaard LS, Borghini Fuhrer I, Kim S, et al. Efficacy and safety of pyronaridine-artesunate for treatment of uncomplicated Plasmodium falciparum malaria in western Cambodia. Antimicrob Agents Chemother. 2016;60:3884-90.

\section{Submit your next manuscript to BioMed Central and we will help you at every step:}

- We accept pre-submission inquiries

- Our selector tool helps you to find the most relevant journal

- We provide round the clock customer support

- Convenient online submission

- Thorough peer review

- Inclusion in PubMed and all major indexing services

- Maximum visibility for your research

Submit your manuscript at www.biomedcentral com/submit 